\title{
Mathematical modeling of an altimeter
}

\author{
Irina Kislitsyna, Galina Malykhina² \\ ${ }^{1}$ Russian State Scientific Center for Robotics and Technical Cybernetics, Saint-Petersburg, Tikhoretsky prospect 21, Russian Federation \\ ${ }^{2}$ Peter theGrate Saint-Petersburg Polytechnic University, Saint-Petersburg, Polytechnicheskaya 29, Russian Federation
}

ABSTRACT

The aim of this survey is to simulate a photon altimeter designed for a soft landing on the lunar surface. A simulation of the process of gamma rays scattering from the lunar surface with a typical composition of the lunar soil was implemented.

\section{Section: RESEARCH PAPER}

Keywords: photon altimeter; lunar soil; gamma rays

Citation: Irina Kislitsyna, Galina Malykhina, Mathematical modelling of an altimeter, Acta IMEKO, vol. 4, no. 4, article 5, December2015, identifier: IMEKOACTA-04 (2015)-04-05

Section Editor: Franco Pavese, Torino, Italy

Received: March 18, 2015; In final form June 15, 2015; Published December 2015

Copyright: (C) 2015 IMEKO. This is an open-access article distributed under the terms of the Creative Commons Attribution 3.0 License, which permits unrestricted use, distribution, and reproduction in any medium, provided the original author and source are credited

Corresponding author: Galina F. Malykhina, e-mail: g_f_malychina@mail.ru

\section{INTRODUCTION}

A photon altimeter is designed for measuring the distance between a lander and the underlying surface. The altimeter should measure the current height of the lander over the lunar surface in the range from $0.3 \mathrm{~m}$ to $10 \mathrm{~m}$ at a descent rate between $6.5 \mathrm{~m} / \mathrm{s}$ and $11 \mathrm{~m} / \mathrm{s}$, providing a random component of the relative error less than $3 \%$. For this purpose a photonic altimeter is developed. The altimeter uses the effect of scattering of gamma radiation over the lunar surface. The conditions of a landing on the moon surface seriously differ from conditions on Earth due to the lack of the lunar atmosphere, a high background radiation and the soil composition of the lunar surface.

There are altimeters of different frequency range such as radio-wave, infrared and optical altimeters, the X-ray and the photon altimeters. The optical altimeters were developed for optical navigation system for lunar landing [1], [2]. We develop the altimeter based on the effect of scattering photons, having the following advantages: the ability to perform measurements through a plasma of engine, the insensitivity to the layer of dust on the surface of the planet, the high measurement accuracy at the low altitudes.

The amount of scattered gamma rays depends on the type of the underlying surface of the Moon [3]. The lunar surface consists of loose material called regolith, fragments of bedrock and secondary particles. The material was formed as a result of continuous meteoric impact and bombardment by atomic particles over billions of years. The average depth of the regolith that covers the entire surface of the Moon ranges from 4 to $5 \mathrm{~m}$ in the lunar seas up to 10 to $15 \mathrm{~m}$ on the continents. The chemical composition of the regolith depends on the composition of rocks lying below, but it also contains other substances and minerals. The main minerals of lunar rocks are plagioclase (solid solution of albite and an orthite $\mathrm{NaAlSi}_{3} \mathrm{O}_{8}$ $\left.\mathrm{CaAl}_{2} \mathrm{Si}_{2} \mathrm{O}_{8}\right)$, orthopyroxene $(\mathrm{Mg}, \mathrm{Fe}) \mathrm{SiO}_{3}$, clinopyroxene $(\mathrm{Ca}, \mathrm{Mg}, \mathrm{Fe}) \mathrm{SiO}_{3}$, olivine $(\mathrm{Mg}, \mathrm{Fe})_{2} \mathrm{SiO}_{4}$, ilmenite $(\mathrm{FeTiO})_{3}$ and spinel-group minerals $\left(\mathrm{FeCr}_{2} \mathrm{O}_{4}-\mathrm{Fe}_{2} \mathrm{TiO}_{4}-\mathrm{FeAl}_{2} \mathrm{O}_{4}\right)$, [4].

Lunar seas are volcanic plains that fill cavities in the topography of the continents. The predominant types of lunar sea rocks are marine basalts. Lunar seas are more suitable for the landing of a spacecraft.

\section{INTENSITY OF SCATTERING PHOTONS}

The altimeter uses the effect of gamma-rays scattering in other words, the Compton effect. The differential cross-section for Compton scattering of one electron per unit solid angle is given by the Klein-Nishina-Tamm formula:

$$
\frac{d \sigma_{\theta}}{d \Omega}=\frac{r_{e}^{2}}{2}\left[\frac{1}{1+\alpha(1-\cos \theta)}\right]^{2} \cdot\left[1+\cos ^{2} \theta+\frac{\alpha^{2}(1-\cos \theta)^{2}}{1+\alpha(1-\cos \theta)}\right]
$$

where $\theta$ is the scattering angle, $r_{e}$ is the classical electron radius, $r_{e}=a r_{c}=\frac{1}{137.04} \frac{\hbar}{m_{e} c} \approx 2.8179 \cdot 10^{-15} \mathrm{~m}$ 
$a=\frac{1}{137.04}$ is a constant

$r_{c}=\frac{\hbar}{m_{e} c}$ is the wave length scattering effect,

$m_{e}$ is the electron mass, $c$ is the speed of light, $E_{\gamma}=660 \mathrm{keV}$ is the energy of the primary radiation, $\alpha$ is the ratio of photon energy to the electron rest energy at $E_{\gamma}=660 \mathrm{keV}$, and $\alpha=\frac{E_{\gamma}}{m_{e} c^{2}} \approx 1.129$.

For a mixture of substances on the lunar surface the effective number $Z_{\text {eff }}$ of the underlying soil surface can be calculated by the following formula:

$Z_{e f f}=\sqrt{\frac{\sum_{i=1}^{M}\left(\eta_{i} Z_{i}\right)^{4}}{\sum_{i=1}^{M}\left(\eta_{i} Z_{i}\right)}}$

where $\eta_{i}$ is the relative quantity of substance $i$, having number $Z_{i}$ of lunar soil, $M$ is the number of components of the underlying surface.

The electron density of the underlying surface is:

$n_{e}=\sum_{i=1}^{6} \eta_{i} \frac{N_{A}}{A_{i}} Z_{i}$,

where $N_{A}$ is Avogadro's number, $A_{i}$ is the atomic mass of substance $i$.

Lunar seas are volcanic valleys filling low places of the continent relief, which are the bottom of large craters and pools. A basalt is a dominant type of the sea's rocks. Composition of the ground shown in Table 1 was used in the model as an example.

The Compton cross-section can be calculated by the equation $\sigma_{0}=6.54 \cdot 10^{-25} n_{e} Z_{\text {eff }}$.

The intensity of the scattered photon radiation $I_{\theta}$ is given by the formula:

$I_{\theta}=I_{0} \frac{3 \sigma_{0}}{16 \pi r^{2}} \frac{\left(1+\cos ^{2}(\theta)\right)}{\left[1+\alpha\left(1-\cos ^{2}(\theta)\right)\right]^{3}}$.

$\left\{1+\frac{\alpha(1-\cos (\theta))^{2}}{[1+\alpha(1-\cos (\theta))] \cdot(1-\cos (\theta))}\right\}$,

where $I_{0}$ is the primary radiation and $\theta$ the scattering angle at a distance $r$ from the scattering electron.

Calculations by (1) to (4) showed that the intensity of the scattered radiation decreases with increasing scattering angle. In the range of angles of interest from $90^{\circ}$ to $180^{\circ}$ the intensity $I_{\theta}$ of a gamma ray of $660 \mathrm{keV}$ does not change dramatically. Therefore, it is advisable to work in this range.

Table 1. Composition of the lunar surface ground.

\begin{tabular}{cccc}
\hline Element & $\begin{array}{c}\text { Percentage } \\
\left(\eta_{i}\right), i=1 \ldots 6\end{array}$ & $\begin{array}{c}\text { Atomic number } \\
\left(Z_{i}\right), i=1 \ldots 6\end{array}$ & $\begin{array}{c}\text { Atomic mass } \\
\left(A_{i}\right), i=1 \ldots 6\end{array}$ \\
\hline $\mathrm{Si}$ & 20.4 & 14 & 28 \\
$\mathrm{O}$ & 41.3 & 8 & 16 \\
$\mathrm{Fe}$ & 13.2 & 26 & 56 \\
$\mathrm{Ca}$ & 0.79 & 20 & 40 \\
$\mathrm{Al}$ & 0.68 & 13 & 27 \\
$\mathrm{Mg}$ & 0.58 & 12 & 24 \\
\hline
\end{tabular}

The energy of the scattered photons depends on the angle of scattering in accordance with the relationship:

$$
E=\frac{E_{\gamma}}{1+E_{\gamma} / m_{e} c^{2}(1-\cos \theta)} .
$$

The photon energy decreases with increasing scattering angle.

If the value of the scattering angle is in the range $\theta=\left[90^{\circ} \ldots 180^{\circ}\right]$, then the energy of the scattered gamma rays reduced down to $200 \mathrm{keV}$. The energy of the scattered photons in the working range depends less on the energy source. The geometry of the gamma radiation source and the gamma radiation detector's location is shown in Figure 1.

The photon radiation source $(S)$ is located in the centre of the measuring system, and four detectors (D1-D4) are placed on a distance $l$ from the source. The lander may be tilted relative to the underlying surface, by an slope angle $\gamma$ of the axis formed by a pair of detectors. Let $h$ be the current height of the landing apparatus, $\psi$ is the angle of incidence of the direct beam of the gamma ray, $\varphi$ is the angle between the projection of the axis of the detectors D1 and D2 on the line passing through the point of incidence, $\theta$ is the scattering angle of photons recorded by the detector D1.

The square of the distance between the scattering element and the detector can be calculated by formula:

$r^{2}=h^{2} \operatorname{tg}^{2} \psi+l^{2} \cos \gamma-2 h l \cos \gamma \operatorname{tg} \psi+(h+l \sin \gamma)^{2}$

The cosine of angle $\varphi$ amounts to:

$\cos \varphi=\frac{(h+l \sin \gamma)^{2}+(h \operatorname{tg} \psi)^{2}+(l \cos \gamma)^{2}-r^{2}}{h l \cos \gamma \operatorname{tg} \psi}$.

The cosine of the scattering angle:

$\cos (\pi-\theta)=\frac{h^{2}\left(1+\operatorname{tg}^{2}(\psi)\right)+r^{2}-l^{2}}{2 r h \sqrt{\left(1+\operatorname{tg}^{2}(\psi)\right)}}$.

The detector registers photons scattered by the lunar surface. The position of the scattering element may be characterized by angle $\psi$ of the direct ray and angle $\varphi$ of the position of scattering element. The range of angle $\psi$ depends on the angle of collimation $\psi_{\max }$, where $0 \leq \psi \leq \psi_{\max }$. The range of angle $\varphi$ is $[-\pi, \pi]$. The distance between the centre and the scattering element is defined by the following formula: $\rho=h \operatorname{tg} \psi$.

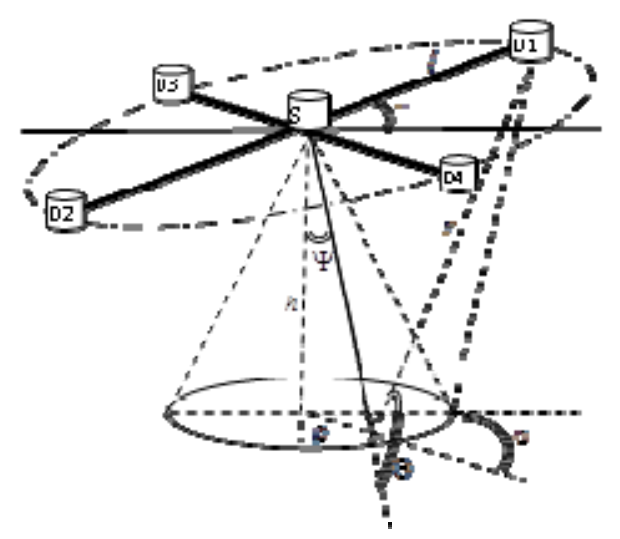

Figure 1. Location of the source and the four detectors. 
The intensity of photons, detected by D1 can be obtained by integration over the scattering volume, depending on the depth, $\varphi, \rho$ :

$$
I_{\text {detect }}=\iint_{\text {depth }} \int_{\varphi} \int_{\rho} e^{-\mu(E) \cdot \rho \cdot d e p t h} I_{\theta}(r, l, \gamma, \psi, h, \varphi, \theta) \cdot d(\operatorname{depth}) d \varphi d \rho
$$

where $\mu(E)$ is the coefficient of mass absorption, depending on the photon energy, $\rho$ is the density of the lunar soil.

The intensity of photons, scattered by an element, situated on the distance $\rho$, the depth (depth) and the angle $\theta$, detected by $\mathrm{D} 1$, is defined by the following equation:

$$
\begin{aligned}
& I_{\theta}(r, l, \gamma, \psi, h, \varphi, \theta)= \\
& =I_{0} \frac{3 \sigma_{0}}{16 \pi r^{2}} \cdot \frac{\left(1+\cos ^{2}(\theta)\right)}{\left[1+\alpha\left(1-\cos ^{2}(\theta)\right)\right]^{2}} \\
& \cdot\left\{1+\frac{\alpha^{2}(1-\cos (\theta))^{2}}{[1+\alpha(1-\cos (\theta))] \cdot\left(1+\cos ^{2}(\theta)\right)}\right\}
\end{aligned}
$$

Detectors D1 to D4 receive the gamma rays scattered from the lunar surface in accordance to the angle of collimation of the radiation source. The detectors register the integral of intensity scattered gamma rays over the volume of interaction with the lunar soil. It is useful to describe the effect of the registration as the ratio of primary and scattered fluxes of gamma rays. The integral of ratio $I_{\theta} / I_{0}$ depends on the angle $\psi$, the depth of penetration of the gamma rays in the lunar soil (depth) and on the height $h$ of the landing apparatus.

\section{ALGORITHM OF MODELING}

The algorithm makes it possible to calculate the ratio $I_{\theta} / I_{0}$ of the registered intensity $I_{\theta}$ and the original intensity $I_{0}$.It consists of three loops for the triple integral computation. The algorithm includes the following steps.

\section{Summary of the algorithm}

1. Initialize the parameters of the model: cross-section for Compton scattering $\sigma_{0}$, ratio of photon energy and energy of electron at rest $\alpha$, mass absorption factor $\mu$, matter density $\rho_{0}$.

2. Set the height $h=0, \Delta h=0.10$, and for $i=1,2, \ldots$, compute the current $h-i \Delta h$.

3. Set the angle of slope $\gamma=0, \Delta \gamma=\pi / 32$, and for $i=1,2, \ldots$, compute the current $\gamma+i \Delta \gamma$.

4. Set the penetration of photons into lunar material, and for $i=1,2, \ldots$, compute the current depth $+i \Delta$ depth .

5. Set the radius of the gamma-ray beam $\rho=0, \Delta \rho=0.10$, and for $i=1,2, \ldots$, compute the current $\rho+i \Delta \rho$.

6. Set the angle of the gamma ray beam $\varphi=0, \Delta \varphi=0.10$, and for $i=1,2, \ldots$, compute the current $\varphi+i \Delta \varphi$.

7. Compute the angle of the gamma ray beam and of the scattering element:

$$
\psi=\operatorname{arctg}\left(\frac{\rho}{\text { depth }}\right)
$$

8. Compute the distance between detector and scattering element:

$$
\begin{aligned}
& r=\left\{\text { depth }^{2} \operatorname{tg}^{2} \psi+l^{2} \cos \gamma-\cos \varphi \cdot 2 \text { depth } \cdot l \cos \gamma \operatorname{tg} \psi+\right. \\
& \left.+(\text { depth }+l \sin \gamma)^{2}\right\}^{\frac{1}{2}} .
\end{aligned}
$$

9. Compute the cosine of the complementary scattering angle:

$$
\cos (\pi-\theta)=\frac{\operatorname{depth}^{2}\left(1+\operatorname{tg}^{2}(\psi)\right)+r^{2}-l^{2}}{2 r \cdot \operatorname{depth} \sqrt{\left(1+\operatorname{tg}^{2}(\psi)\right)}} .
$$

10. Compute the elementary scattering volume:

$$
\Delta V=\Delta h\left(2 \Delta \rho \cdot \rho+(\Delta \rho)^{2}\right) \cdot \Delta \varphi .
$$

10. Compute the accumulation of the gamma intensity ratio:

$$
\begin{aligned}
& \frac{I}{I_{0}}=\frac{I}{I_{0}}+\frac{3 \sigma_{0}}{16 \pi r^{2}} \cdot \frac{\left(1+\cos ^{2}(\theta)\right)}{\left[1+\alpha\left(1-\cos ^{2}(\theta)\right)\right]^{3}} \\
& \cdot\left\{1+\frac{\alpha(1-\cos (\theta))^{2}}{[1+\alpha(1-\cos (\theta))] \cdot(1-\cos (\theta))}\right\} \cdot \Delta V .
\end{aligned}
$$

12. Compute the gamma intensity ratio subject to the absorption of photons:

$$
I=I_{0} e^{-2 \mu \delta \cdot d e p t h} .
$$

The mass absorption factor $\mu$ of photons with energy $660 \mathrm{keV}$ by silicon equals $\mu_{1}=0.0802 \mathrm{~cm} / \mathrm{g}^{2}$. The mass absorption factor of the scattering gamma-quantum with energy $200 \mathrm{keV}$ by silicon equals $\mu_{2}=0.123 \mathrm{~cm} / \mathrm{g}^{2}, \delta$ being the density of the matter.

\section{NUMERICAL EXPERIMENT}

The proposed model is implemented as a simulation program running in Matlab. Dependences of the average intensity of scattered gamma-ray quanta detected by D1 on a height of the descending module are presented in Figure 2.

The gamma-ray flux-noise is characterized by a Poisson distribution. Therefore, the result of measuring the height and the angle actually is not a smooth function of the intensity, as shown in Figure 2. Noisy data registered by the four photon detectors should be averaged. Figure 3 shows the dependences of the height on the intensity of photon flux for different values of the angle. The measurement result is the average height of the four outputs of the detectors.

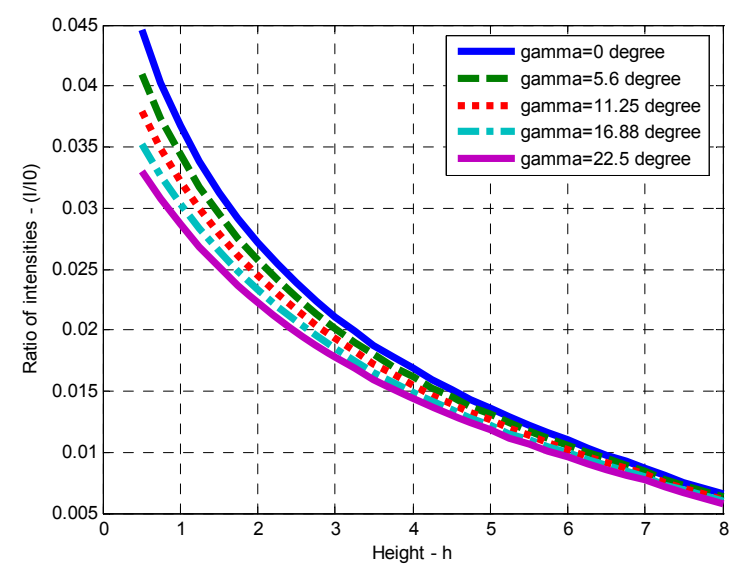

Figure 2. The ratio of average intensity of scattered and initial gamma quanta versus the height of the descending module. 


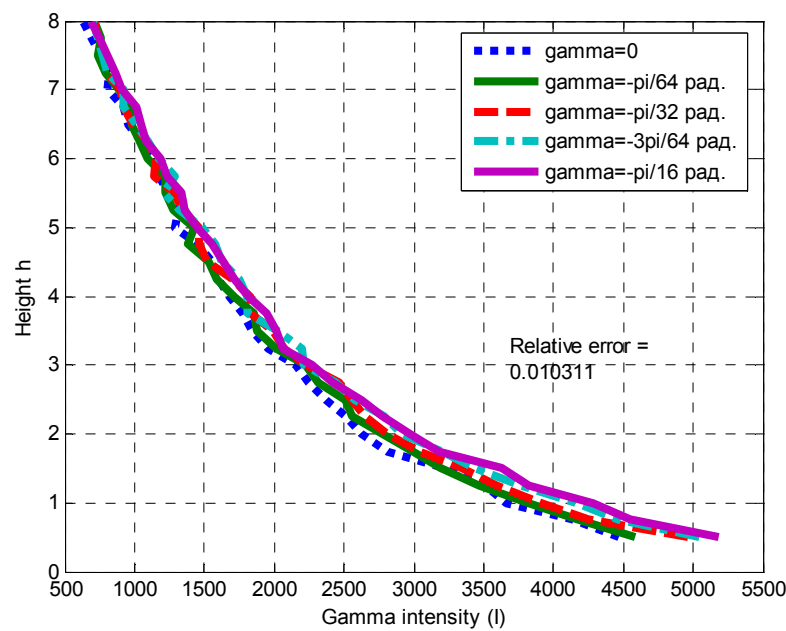

Figure 3. Dependences of the height on the intensity of photon flux for different values of the angle.

Each detector records the angle of the lander.Dependences of the measured angle on the height computed using the intensity of the gamma-ray flux are shown in Figure 4.

The signal received by the detector is stronger when the height of the descending module is small. The height and slope angle can be measured more accurately when the height is small. This fact is an advantage of the photon altimeter. It is more difficult to measure height and slope angle at high-altitude of the descending module.

The results of the altimeter model showed that the standard deviation of error of slope angle for low-altitude of the descending module is not greater than $0.02 \mathrm{rad}$. The standard deviation of the error of the slope angle for high-altitude of the descending module is less than $0.06 \mathrm{rad}$.

\section{CONCLUSIONS}

The proposed model is based on initial values:initial height,activity of photon source, topology of photon sources and detectors, composition of the ground.

The developed model allows estimate effect of the topology of the measuring system, activity of the source, size and arrangement of the detectors, the composition of the ground.

The model allows determine basic characteristics of altimeter with reasonable accuracy. The range of measurement of

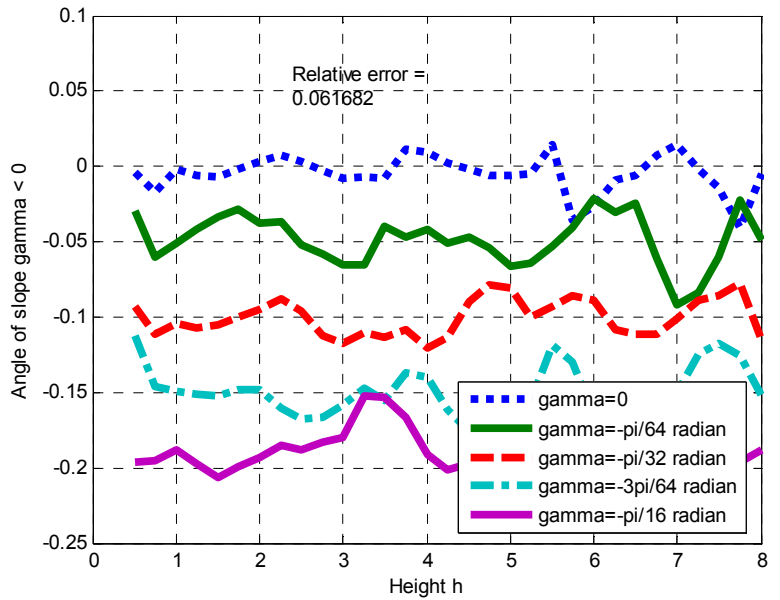

Figure 4. Dependences of slope angle measured for different heights.

altitude, the effect of landing apparatus slope, the relative error of measurement may be estimated.

The model submit for consideration capability of angle slope measurement.

\section{ACKNOWLEDGEMENT}

The scientific research was supported by Central Research Institute of Robotics and Technical Cybernetics. Authors are grateful for scientific and financial support.

\section{REFERENCES}

[1] V. Simard Bilodeau, S. Clerc, R. Drai, J. de Lafontaine, Optical Navigation System for Pin-Point Lunar Landing, Preprints of the 19th World Congress The International Federation of Automatic Control Cape Town, South Africa, August 24-29, 2014, pp. 10535- 10542.

[2] Xiangyu Huang, Dayi Wang, Yingzi He, Yifeng Guan, Autonomous Navigation and Control for Pin point Lunar Soft Landing, 7th International ESA Conference on Guidance, Navigation \& Control Systems 2-5 June 2008, Tralee, County Kerry, Ireland.

[3] E. I. Yurevech, Photon technique, Saint-Petersburg, press of the Saint-Petersburg Polytechnic University, 2003, 235pp, (in Russian).

[4] V.P. Legostaev and V.A. Lopota Moon-a step towards technology development of the solar system. / M.-RSC "Energy", 2011. 684pp, (in Russian). 